\title{
Research on Localization Strategy of MCN Short Video
}

\author{
Ying Shi, Bo Zhang, Can Li, Guifu Li \\ Shanghai Publishing and Printing College, Shanghai, China \\ Email: syfanxiang@163.com
}

How to cite this paper: Shi, Y., Zhang, B., Li, C., \& Li, G. F. (2020). Research on Localization Strategy of MCN Short Video. Open Journal of Social Sciences, 8, 411-418. https://doi.org/10.4236/jss.2020.85028

Received: April 16, 2020

Accepted: May 26, 2020

Published: May 29, 2020

Copyright ( 2020 by author(s) and Scientific Research Publishing Inc. This work is licensed under the Creative Commons Attribution International License (CC BY 4.0).

http://creativecommons.org/licenses/by/4.0/

\begin{abstract}
MCN (multi-channel network) is the product form of multi-channel network. It was born in 2009 on the YouTube video platform in the United States. In 2013, the new film was introduced to China and launched in 2015. The market environment of China is different from that of the United States. The MCN model has traveled across the ocean to China, and a series of changes have taken place in the soil of China. This paper aims to clarify the development and evolution of the new concept of MCN in China, as well as the problems and countermeasures in the process of localization.
\end{abstract}

\section{Keywords}

MCN, Short Video, Localization

\section{Introduction}

MCN is an imported product, which is a product form of multi-channel Network. It combines PGC content and guarantees the continuous output of content under the strong support of capital, so as to realize the stable realization of business eventually. MCN mode originated from the mature web celebrity economic operation abroad, and its essence is the product form of a multi-channel network. It combines the content of PGC (professional content production) and guarantees the continuous output of the content under the strong support of capital, so as to realize the stable realization of the business finally.

This paper mainly describes the development, evolution and localization problems of MCN mode after it is introduced into China's short video field, and discusses the solutions to the problems in the development process. Through the research on the development status of MCN mode in foreign countries and the preliminary practice in China, the localization status and problems of this de- 
velopment mode are studied, the necessity of the existence of this mode and the prospect of this development mode are explored, so as to provide directional guidance for practice.

This paper mainly adopts the text analysis method, through the MCN mode in the short video field at home and abroad of the development of data collection, comparison, analysis of the problems in the process of localization development and the future development direction.

\section{Research on the Development Status of Domestic MCN Model}

At present, there is no clear definition of MCN (multi-channel network). As an imported product, MCN has flown across the sea to China. Due to the different market and the rapid change of short video industry, the concept of MCN has shifted. The general consensus in the industry is that companies with the ability and resources to help content producers can be called MCNS. To be specific, MCN will generally, based on its understanding of the market and brand partners, be deeply involved in the production and release of CP content to help its content better adapt to the market environment. From the perspective of the platform, MCN is regarded as the direct partner and new realization means after the collapse of the "platform-cp" secondary structure, and the platform distributes appropriate traffic and corresponding population to it. In simple terms, it means that an organization distributes content on a content platform through numerous accounts. Extended meaning can be understood as: an intermediary that can serve and manage a certain number of accounts.

In recent years, the comprehensive guarantee of technology and the improvement of audiences' media literacy have provided an opportunity for MCN to take root and grow rapidly in China.

\subsection{Reasons for the Rapid Development of MCN}

The development of mobile technology has laid the foundation for the rapid spread of MCN. "Short video" is compared with the traditional "long video", most of which is less than 5 minutes long. It is mainly transmitted by mobile intelligent terminals and Shared on social media platforms. The widespread popularity of smart phones enables people to watch short videos regardless of time and place; the development of $4 \mathrm{G}$ network provides technical support for high-quality data transmission; and the coming era of $5 \mathrm{G}$ is about to bring unpredictable development situation. The rise of social network increases the stickiness of users, the fragmented reading habits of users meet the needs of users, and the joining of giants promotes the influx of industry capital. These reasons contribute to the rapid development of short videos.

A large number of UGC Generated Content producers have also become the audience market of short video transmission. Data from iresearch in the first half of 2019 shows that $71.7 \%$ of users of the vertical short video APP have used 
the function of shooting and making short videos. At the same time, UGC short video web celebrity attracts a large number of fans, drives a series of fans economy, and becomes a new growth point for the development of short video. However, the low average video quality and weak continuous liquidity of individual short video creators become constraints for their development, thus the MCN organization emerges at the right moment. MCN organization is to combine the scattered PUGC (PGC + UGC + exclusive copyright) to ensure the continuous output of content, so as to eventually realize the stable realization of business, and become the booster of short video development (Qian, 2018).

\subsection{Features of MCN Project}

The value provided by MCN can be roughly divided into two categories: information value and efficiency value.

MCN projects with investment value, they have: strong content judgment and production capacity; It is better to have their own template, industrial production mechanism; at the same time, it has a strong capacity to purchase the flow, and has the control over the flow like a faucet. It can realize the regulation from both sides of content production and consumption and form a match. At the same time, the service ability of the company to the red people is also very important, to let the red people willingly store, Ma Nanshan, do a pure content producer, so as to produce the ability to control the red people, which requires a very detailed and systematic service.

MCN's vertical choice is also worth talking about. When the video market has developed to this extent, for MCN, the most realistic and most recognized model in the capital market must be to deepen in a vertical field and then extend the successful experience to more market segments. For example, the old MCN Awesomeness TV is cut from teenagers, and the recently popular Taste made is cut from food. In terms of the entry difficulty, the choice of some unpopular fields will guarantee the strong negotiation ability and the rapid expansion of the traffic to the popular people, but it also brings a problem: the value of the traffic in the unpopular fields is very low, and the realization of advertising and e-commerce will encounter many obstacles.

\subsection{The Mode of MCN Localization Development}

After the introduction of MCN mode into China, according to the development of the Chinese video industry, the source and way of its localization development can be divided into three different types: MCN based on the short video platform gene, MCN based on the vertical domain MCN based on the content and MCN based on the matrix MCN based on the header web celebrity/IP.

MCN based on short video platform gene. Short video platform based on its own professional advantages and content gene, to MCN is a new attempt of its development and transformation. In 2012, relying on the short film sharing platform, the new film market was established and gradually developed into a 
leading domestic new media film production and distribution platform. At the end of 2013, the MCN concept was first proposed by the new film studio in China, and this model was brought to China through in-depth visit and research of Maker Studios in the United States. In 2015, the new film market invested in the brand of "creation collection" and put these short videos on the channel, which gained 150,000 fans in a week and 700,000 fans in a month. Next, the new film field began to force the original video brand. The most typical is the magic TV series, such as "magic food", "magic travel", "magic mode" and other short videos. These original short video advertising business agent, brought a lot of revenue, and, precipitation under many own IP. While perfecting the original short video matrix, the new film also signed a large number of popular KOL. In 2015, the new film field tried to cooperate with the MCN we media channel of Sohu video. After more than half a year, the new film field became the largest MCN organization of Sohu, with more than 150 content brands. In April 2016, the new film released the magic box, followed by the release of the series of magic programs "magic food", "magic mode", "magic TV" and other channels. In the view of Yin Xingliang, CEO of the new film field, "only for the project to do traffic, promotion and other aspects of support do not conform to the new film field of content gene, do a video with its own trump MCN is the goal." (The Investment Community, 2017).

Content-based vertical domain MCN. "Vertical" has become the only way to segment the market, and is increasingly seen as a cure for MCN. From abroad, Awesomeness TV, which focuses on children's video content, is considered to be a platform with clear brand value, stable traffic, high user stickiness and conversion rate without the head web celebrity, and easier to be favored by advertisers. In the past two years, Maker Studios, Full screen and Network have also started to build their own content IP. In China, a group of teams that previously focused on vertical content production, after developing the MCN model, completed the transition from content production to MCN based on the professional degree and operation ability accumulated for vertical content. One example of this type of vertical content MCN is the ivy culture. Since 2014, Sanyo culture has transformed from a video production company to an original content production company, and then developed two content IP's, namely, understanding mom (in the field of maternal and child) and bump and bump (in the field of quadratic elements). Based on this, the green ivy derived from the mother and baby, secondary and pan-lifestyle vertical areas such as MCN business.

Matrix MCN based on header web celebrity/IP. Start with a hot IP, and then sign up potential account, become a lot of web celebrity /IP expansion path. Around April 2017, Papitube, Jiang's team, signed up nearly 30 short video creators, making Papitube a veritable MCN. Papitube's MCN model follows a set of "assisted nurturance" models: new content is streamed through the head account's traffic and attention, and operations, content, distribution and moneti- 
sation strategies, known as "big with small". For example, Papi Jiang will forward short video creators incubated by MCN on Weibo or WeChat. Through Papitube's operation, several accounts with more than one million followers or close to one million have been hatched. In the future, the contracted short video creators under Papitube will conduct vertical operation of we media along the direction of lifestyle, and finally create a vertical we media matrix of life.

\subsection{The Industrial Pattern of Domestic MCN}

In recent years, the short video industry has been booming, attracting a lot of capital and attention from all sides. The status quo of MCN's localization industrialization pattern can be summarized in two parts: the first part is the active layout of the domestic Internet giants; the second part is the overseas MCN organizations that rush into the Chinese market.

Active layout of domestic Internet giants. Domestic Internet giants and video production platforms have made frequent efforts in the short video industry to promote the development of MCN by investing in video platforms, integrating resources, motivating content creators and optimizing management mode. According to Quest Mobile newly released data, in June 2017, as user size, seconds video shoot, well quickly, potatoes, watermelon, beauty industry ranked the top five, top trend clear: "second shot-Weibo" system (SEC making/small cafe/sway cafe), today's headline (volcanic small video/video/watermelon trill), well quickly. In 2018, Weibo will set up a 3 billion yuan joint production fund to deeply support MCN organizations that produce high-quality content in the fields of content e-commerce, high-quality short videos and pan-entertainment from the aspects of capital, creativity and commercialization (Jiang, 2019). Toutiao launched 1 billion support short video plan, Miaopai took 1 billion to launch the video creator platform, Youku set up 3 billion PGC industry fund. In addition, BAT also actively entered the market: Tencent launched grain in ear plan 2.0, which plans to invest 1.2 billion yuan to support We media, of which 1 billion yuan will be focused on original and short video authors. On the basis of 1 billion yuan content fund, Alibaba plans 1 billion yuan cash subsidy for video authors. Baidu set up a 500 million yuan content investment fund and invested in Renren video.

Overseas MCN in the Chinese market. China's short video red sea is also attracting increasing attention from overseas MCN companies. Among the pioneers, the Netherlands' MCN zoomin.tv was one of the first to "rush for gold" in China, having set up shop there in April 2016. As the largest MCN in Europe, zoomin.tv has 50,000 we-media channels and 150 million followers. After entering China, zoomin.tv first carried out a large-scale action, which was to sinicize the video content that performed well in foreign countries, and then released it on various short video platforms, and introduced domestic short videos, most of which were exotic and adventurous videos. Yoola, from Israel, is also exploring the Chinese market. Now, Yoola has started to open accounts and launch opera- 
tions for a group of foreign celebrities on various domestic platforms. The first batch of short video bloggers to be introduced to China are Sliviki Show, Room Factory and Roman Ursu, two of which are popular science and the other is funny.

\section{MCN Localization Development Strategy}

The progress of technology, the development of the market and the influx of capital promote the rapid development of MCN model after entering China, which has become a new booster for the development of short video. Faced with the market characteristics of China, diversified distribution platform, multi-directional development mode and multi-channel profit mode make it necessary to localize the imported product MCN. Its development direction can be attempted from the concept evolution, channel expansion, vertical subdivision of content, and diversification of profit mode.

\subsection{The Evolution of the Concept-From MCN to MPN to MBN}

Since the initial introduction of MCN, this concept has been mutated in China. The short video market in China is crowded, different from the dominant one of foreign YouTube, MCN enters China with the nature of MPN (multi-platform network) multi-frequency Platform Network distribution. On the one hand, all kinds of institutions continue to throw out the MCN flag, and get the attention of capital and large platforms; On the other hand, all kinds of warnings were kept, "the development of MCN in China cannot copy the overseas YouTube model, after all, the Platform and ecological difference are so huge", so there are also MPN (multi-platform Network) multi-frequency Platform Network management, MBN (multi-branding Network) multi-brand content management. As an upgraded version of MCN, MPN is focused on the broadcast channel is not a single, that is, multi-platform distribution. In view of the influence of various domestic video platforms, the concept of MPN is more suitable for the current market situation. MBN is a concept created by the new film field, that is, multi-branding Network, which mainly wants to focus on brand management of content. Because different from foreign video platforms, the domestic MCN business model needs to explore more suitable for local diversified realization methods-advertising, e-commerce, IP, web celebrity economy and so on.

\subsection{The Expansion of Channels-Diversified Distribution to Ensure the Scope of Transmission}

The major domestic video sites are equal in strength, to ensure the maximum dissemination range to achieve the best dissemination effect, all must carry out multi-platform distribution mode. Papitube, for example, since it was established in April 2016 Papitube, makes a content delivery across multiple platforms, including video platform Youku video, iQIYI video, Beep Beep Mile Miles, such as social platform Weibo, WeChat public number, etc., after more 
than a year of development, Papitube there are signing author more than 30 , Weibo fans of total quantity is 37 million, Weibo last year total amount of powder more than 10.31 million a year, the whole platform always play 11 billion.

\subsection{Vertical Subdivision of Content-Specialized Division of Labor to Ensure Intensive Farming}

"Vertical" is increasingly seen as a cure for MCN. Overseas, Awesomeness TV, which focuses on children's video content, is regarded as a model in the vertical field, with its clear brand value, stable traffic without the head web celebrity, high user stickiness and conversion rate, and easier to be favored by advertisers. From the perspective of China, a group of teams previously focused on vertical content production developed the MCN model, and based on the accumulated expertise and operational ability of vertical content, they signed horizontal contracts and incubated other content parties in the vertical track to ensure the stable development of vertical content.

One example of this type of vertical content $\mathrm{MCN}$ is the ivy culture. Since 2014, Sanyo culture has transformed from a video production company to an original content production company, and then developed two content IP's, namely, understanding mom (in the field of maternal and child) and bump and bump (in the field of quadratic elements). Based on this, the green ivy derived from the mother and baby, secondary and pan-lifestyle vertical areas such as MCN business. Earlier, ivy culture CEO Ji Fangyuan said in an interview with Quanmediaist, "we plan to sign up or incubate $60 \%$ of the top 50 maternal and child accounts on Weibo in 2017." In Ji's opinion, the truly valuable "vertical field" not only needs to have a vertical crowd, but also needs to have a vertical market, that is, the audience of the content and the consumer market highly coincide, after reading the content can directly affect the consumption. MCN shares its accumulated resources to allow contracted content creators to speed up the discovery process.

\subsection{The Diversification of Profit Mode-Corporate Operation Ensures Stable Commercial Realization}

As a professional company, MCN has strong management strength and liquidity. Through the aggregation of PUGC contents, it has formed a strong bargaining power and a more diversified way of profit. At present, there are three common ways to cash: one is vertical upward, from short video to network drama, to the big movie; Second, vertical downward, to charge for content, to e-commerce, to community economy; Third, horizontal expansion, systematic content replication or KOL production. The first two roads are very difficult to get through. The representatives of the two roads are wan Hetian Yi and Luo Ji thinking. "For more PGCS, horizontal scaling is the most affordable option for their traffic growth and capital story, with or without a header." (Wang, 2017)

In terms of diversified distribution approaches, different platforms have their 
own optimal profit modes. What MCN needs to do is to take measures according to local conditions, adopt diversified profit modes, and play a combination punch to achieve the maximization of benefits ( $\mathrm{Li}, 2019)$.

\section{Summary}

To sum up, MCN, an imported product, must implement localization strategy in China's short video industry, and its development direction can be started from four aspects: concept change, channel change, operation mode change and profit mode change. At present, in order to ensure that the MCN model can truly take root and sprout in China, efforts must be made from the following four aspects: first, diversified distribution should be carried out to guarantee the spread range; second, mutual linkage should be ensured through cluster development; third, intensive farming should be ensured according to the specialized division of labor; fourth, stable realization of corporate operation should be ensured.

\section{Funding}

This paper is the research result of the art and science planning project of Shanghai in 2019, project No. YB2019C02.

\section{Conflicts of Interest}

The authors declare no conflicts of interest regarding the publication of this paper.

\section{References}

Jiang, Z. Z. (2019). Problems and Countermeasures of MCN Platform Development in China. New Media Research, 23, 66.

Li, J. Y. (2019). Research on the Development Mode of MCN in Short Video Industry. Research on Transmission Competence, 25, 126.

Qian, Y. B. (2018). The Localization Development of MCN Model. Journal of News Research, 13, 94

The Investment Community (2017). WEB Since 2017 Media Revenue of 20.7 Billion Yuan Will Set Up 3 Billion Fund to Support MCN. http://pe.pedaily.cn/201712/424105.shtml\#jtss-tsina.

Wang, W. B. (2017). Short for Good-Analysis of the Development Status and Trend of Short Video in China. TV Research. 